\title{
HITOS PARA UN ITINERARIO: LOS NOMBRES DE LUGAR EN
} LA PEREGRINATIO EGERIAE

MILESTONES FOR AN ITINERARY: PLACE NAMES IN THE PEREGRINATIO EGERIAE

\section{RESUMEN:}

Abstract:

Peregrinatio Egeriae es un manuscrito que Peregrinatio Egeriae is a manuscript which narra el viaje de Egeria, una mujer del siglo tells the journey of Egeria, a woman from IV. El documento está escrito a modo de diario 4th century. The document was written as a de viaje, por lo que proporciona multitud de travel diary; therefore, it provides with many detalles. Este artículo tiene como objetivo details. This article aims to go deeper into the profundizar en el es en el estudio de los topónimos study of the place names which appear in this que aparecen en este manuscrito manuscript.

\section{Palabras claves:}

Peregrinatio Egeriae, topónimos, Egeria.

\section{A Alicia, in memoriam}

La Peregrinatio Egeriae recoge el relato de un viaje realizado a finales del siglo IV por una mujer y cuyo destinatario es un grupo de mujeres que la esperan en el lugar de origen de la primera y a las que se refiere con gran afecto y cariño. Se encuentra concentrado en un solo manuscrito hallado en 1884 por G. F. Gamurrini en la ciudad italiana de Arezzo. Es un manuscrito incompleto, ya que le faltan el principio y el final así como algunas páginas en el centro 1 . No obstante, se han encontrado varios fragmentos que han ayudado a completar alguna de esas lagunas. Por un lado, los fragmentos que encontró el Padre De Bruyne (1908:481-484), en un manuscrito del siglo IX conservado en la Biblioteca Nacional de Madrid y por otro, el nuevo fragmento encontrado por el editor Jesús Alturo en una colección privada de Madrid y que podrían pertenecer al ejemplar, hasta ahora perdido, del Monasterio de San Marcial de Limoges (Alturo, 2005:241-250). La parte conservada la podemos dividir en dos partes: en la primera se trata efectivamente de la narración de una serie de viajes de peregrinación por los lugares más significativos de Tierra Santa, así como de los lugares bíblicos y monásticos del Oriente cristiano. La segunda narra exhaustivamente las celebraciones litúrgicas de Jerusalén, partiendo de la descripción de los actos cultuales diarios y terminando con la organización completa del año liturgia. Se trata de una fuente fundamental para el conocimiento del culto en la Jerusalén de finales del siglo IV.

Sabemos muy pocas cosas de la autora de la Peregrinatio.

Del contenido del Itinerarium podemos deducir algunos rasgos de la persona que lo redactó: su sexo, alguna referencia a su procedencia (en el otro confín del mundo: "ut de extremis terris uenires ad haec loca"-19.5-), a su posición socioeconómica y de su personalidad. Desde la aparición del manuscrito, aquella persona que se ha acercado a él ha planteado su hipótesis acerca de quién pudiera ser la autora, porque de lo que no hay ninguna duda es de que es una mujer. El mismo Gamurrini, en 1885, propone como autora a una tal Silvia de Aquitania, hermana del prefecto de Pretorio del emperador Teodosio I entre el 383 y el 395, Flavio Rufino² (Gamurrini, 1885:158159). La única prueba que aduce es la intuición: "Mi apparve come improvviso un raggio" (Gamurrini, 1885:158). Köhler en 1884 hizo otra propuesta: Gala Placidia, la hija de

1 En concreto el primer y el último folio del segundo cuaderno. Para una discusión en profundidad sobre este punto, las vicisitudes del manuscrito y las principales ediciones y traducciones, cfr. Cruz (2003:105-123).

2 Tres años más tarde, le surgirán algunas dudas y las primeras precisiones. Silvia no es la hermana, sino la cuñada de Rufino y hay dudas de que fuera de Aquitania. Aunque, en efecto, esta Silvia, cuñada del prefecto, existió y viajó a Tierra Santa en el 396 junto a su hermana viuda -Rufino había sido asesinado el 27 de noviembre de 395 (Arce 1980:18-19).-y su sobrina. Durante cuatro años visitò Palestina y Egipto. Las fechas de su viaje, del 396 al 400 (Arce 1980:18-19 y Arias 2000:10, n.4), hace que sea imposible que fuera la autora de un texto datado, casi con toda seguridad, entre el 381 y el 384. De todas formas, la consulta de Gamurrini de la Historia Lausiaca de Palladio debió de ser bastante apresurada, ya que en él se habla de una tal Siluania, no Silvia (Cardini 1989:43). 
Teodosio (Köhler, 1884:141-151), pero dado que nació en torno al 388 es muy poco probable que pudiera ser la autora de un viaje realizado antes de que ella naciera (Arce, 1980: 19).

En 1903, Ferotin publica un artículo en la Revue des Questiones historiques con lo que hasta hoy es la hipótesis más probable de autora, al relacionar el manuscrito de Gamurrini con un escrito del monje de la segunda mitad del siglo VII, Valerio del Bierzo (Ferotin 1903:367-397). Este escrito, una carta cuyo título completo es Epistola de Beatissimae Echeriae laude ${ }^{3}$, era bien conocido desde el siglo XVIII, siendo incluido por Flórez en su monumental España Sagrada. Esta carta es un homenaje ("lauda"), a una santa mujer ("beatissima"), llamada Egeria que viajó desde un extremo del mundo al otro, animada por un celo religioso y por el afán de conocimiento. El recorrido de Tierra Santa que hace la carta y el Itinerarium coinciden casi punto por punto. Se han propuesto diferentes variantes a la grafía (Natalucci, 1999:44-45), del nombre de esta mujer: Egeria, Eucheria, Echeria, Etheria, Aetheria,..., que se deben, sobre todo, a las dificultades de lectura de los códices. Nosotros seguimos la forma más habitual, Egeria que, además de ser la más popular, es la que podemos confirmar en diferentes fuentes anteriores, contemporáneas y posteriores al Itinerarium.

En ese mismo año, Bouvy (1903:514-522; 1904:80-83) propone como autora a una tal Eucheria, hija del conde Eucherius, tío paterno de Teodosio y cónsul en 381. Bouvy no pone en duda la autoría dada por Ferotín, sino la identificación del personaje descrito por Valerio. Investigaciones posteriores, sobre esta Eucheria, han hecho que se abandonara esta hipótesis (Arce, 1980:19-20). Pues, a pesar todo el valor que tiene el texto de Valerio, del personaje de Egeria no proporciona muchos más datos.

El otro gran dato que podemos entresacar de la carta de Valerio es el punto de partida del viaje, y por tanto, la nacionalidad de la autora. Todo el debate se centra en el estudio de dos frases. Una del Itinerarium (19.5): "ut de extremis porro terris venires ad haec loca". Y otra de la carta de Valerio (n. 4): "extremo occidui maris Oceani litore exorta, Orienti facta est cognita". La carta no deja lugar a dudas, Egeria "parte de" o "nace", según la traducción que se haga del verbo exorta (n.4), del extremo occidental del mar Occidental, lo que tradicionalmente se conoce como Galicia. La expresión de extremis terris tiene dos sentidos (Cardini 1989:43).: una genérica, que, en boca de una persona de Oriente, podría hacer referencia a todo el Occidente; y otra más restringida, que

3 Es tradicional que la carta acompañe a las traducciones hechas del Itinerarium, bien como apéndice, bien como parte de la introducción. De ellas solo la obra de Arce (1980) y Janeras (1986) nos ofrecen la versión latina, acompañando a la traducción. La edición príncipe de esta carta se encuentra en los Anales del reyno de Galicia II, publicada en 1736 por Francisco Xavier Manuel de la Huerta y Vega en Santiago de Compostela (pp. 379-381). No obstante, el valor de la publicada por Flórez reside en el hecho de haber hecho una completa revisión de los cuatro códices más conocidos hasta el momento el de Carracedo, el de Toledo, otro códice de Toledo del s.XII y el de San Millán de la Cogolla (Arce 1980: 4-17). reduce el Occidente a una región muy concreta: el área de la península Ibérica cercana al océano Atlántico.

Como es obvio a cada una de las identificaciones anteriores de una autora, se corresponde un origen o patria diferente. La gran competidora de Hispania es la Galia habiendo hecho originaria a la autora del Itinerarium tanto de Aquitania (Gamurrini, 1887;Meister, 1909:363-368;Trillitzsch, 1971:104-111) o de la Galia Narbonense, al sur de la Galia, como de Normandía (Weber, 1989:437-456), al norte. Como en el caso de la identidad de la autora, nos decantamos por la hipótesis más popular (Arias, 2000:13 14), esto es, como gallega.

Viajar de un extremo del mundo a otro no debía de resultar ni fácil ni barato. A Egeria se la recibe con los brazos abiertos y en algunas ocasiones goza de escolta imperial, cuando la naturaleza de la ruta así lo aconseja. Las dificultades para organizar un viaje de este tipo (transportes, comidas, alojamientos,...) eran muchas y Egeria hace veladas alusiones a ellas, aunque sin aportar muchos datos, como si fuera algo normal. Y lo normal no es lo que se comunica en una carta. Todo ello nos revela a una dama de posición más que acomodada, con una cierta influencia política.

Pero quizá lo que nos fascina del Itinerarium es lo que no revela sobre la personalidad de su autora. Sabemos que viaja sola, que obispos y presbíteros se desviven por ayudarla y mostrarle los lugares que desea visitar, que no tiene problemas económicos y que tiene un gran conocimiento de la Biblia. Ahora, ¿quién era esta Egeria? ¿Una monja, una mujer consagrada a Dios, que formaba parte de una comunidad con la que se mantenía en contacto, a través de una correspondencia epistolar, de la que el texto del que hablamos era solo una muestra? $\mathrm{O}$, ¿era, más bien, una dama piadosa y adinerada que viajaba animada por su devoción y por su afán de conocimientos? Y si es este el caso, ¿qué relación tiene con las mujeres a las que escribe?

Son muy pocos los datos que el manuscrito de Gamurrini nos aporta acerca de las destinatarias del libro de viajes. Solo unas cuantas menciones a lo largo del texto en forma de vocativo. En ellas Egeria se refiere a las destinatarias como dominae uenerabiles sorores $(3.8,20.5)$, affectio uestra $(5.8,7.3,20.13,24.1,27.2)$, dominae uenerabiles $(12,7)$ dominae animae meae $(19,19)$, domnae, lumen meum $(23,10$, dos veces), y dominae sorores $(46.1,46.4)$. De todo ello, se deduce una relación muy estrecha sobre todo desde el punto de vista afectivo, que ha llevado a afirmar la pertenencia a una comunidad monástica de vírgenes. La carta de Valerio del Bierzo que la nombra como santimonialis, palabra que es al latín medieval, lo que hoy entendemos por monja (Arce, 1980:69).

Jerusalén, Sinaí, Etam, Tatnis, Dennaba, Jordán, Jericó, Ur, Antioquía, Capadocia, Constantinopla... son algunos de los topónimos que aparecen en la Peregrinatio Egeriae. Algunos son muy conocidos. Otros nos sonarán por motivos de lo más diverso. Etam 
nos recuerda a una conocida marca de lencería femenina. Tatnis es la ciudad en la que Indiana Jones encuentra el Arca Perdida. Jerusalén, Sinaí o Jericó suelen aparecer en los telediarios con noticias de diferente carácter. Capadocia es un destino turístico bastante frecuente, aún hoy día entre quienes visitan Turquía. Y Constantinopla ha cambiado su nombre romano por el turco Estambul. Son nombres de lugar, topónimos que están presentes en nuestra realidad aún hoy día. Pero en época de Egeria no eran tan conocidos. Es más, algunos son conocidos por primera vez gracias a ella, como Clesma, Carneas, Mansocrenas, o Pithom (Milani, 1969:213-214). Pero todos ellos forman el eje articulador en torno al que Egeria construye su viaje y en último término, su relato.

Siguiendo la definición de De Felice (2007:164), deberíamos señalar que vamos a estudiar todos los topónimos del área comprendida desde Constantinopla hasta Alejandría, y desde el Mediterráneo hasta el Éufrates, en el arco temporal, de la segunda mitad del s. IV. Dado que nuestro objetivo es conocer en profundidad una obra del s. IV, hemos introducido una tercera variable en la definición clásica de De Felice: la circunscripción a una obra literaria concreta. Nuestro objetivo será pues estudiar los topónimos que utiliza Egeria para saber si son términos latinos, griegos, y hebreos, tomados de la Biblia,...

\section{Repertorio toponímico}

Como hemos visto, Egeria viaja por todo el Cercano Oriente. En ese viaje recorre una serie de lugares, algunos reales, como Jerusalén, otros míticos, como la Tierra de Gessén, que solo aparece en la Biblia. Los lugares que visita son el repertorio del que nos vamos a ocupar. Hemos identificado un total de 86 topónimos, que listamos a continuación, tal y como los menciona el texto latino.

Agri specula,
Alexandriam,
Anthiocia,
Arabia
Arabot, montem
Asia
Augustofratensis, prouinciae
Ausitidem, regionem
Basan
Batanis
Belsefon
Bithiniam
Calcedona
Cappadociam
Carneas
cepos tu agiu Iohanni (hortus Sancti Iohannis)
Chanaan, terram
Charris (Charra)

Clesma

Constantinopolim

Corico

Corra

Dennaba

Edessam

Edom

Efesum

Egyptum

Enon

Epauleum

Esebon (Exebon)

Eufraten

Fadana

Faran

Feniciis, partes

Fogor

Galatiam

Gerapolim (Ierapolim)

Gesse, terra

Helia

Hero (Heroum ciuitas)

Hisauria

Hur

Idumeae

lericho

Ierusolima

Iordanem

Israhel

Libiada

Magdalum

Mansocrenas

Memoriae concupiscentiae

Mesopotamiam

Mesopotamiam Syriae

Moab, terra

Mortuum, mare

Nabau, montem

Nili

Nisibin

Opu Melquisedech

Palestinam

Parthenicum, mare

Pelusio

Persida

Pithona

Ponpeiopolim

Ramessen

Rodanus

Rubrum, mare 


Safdra
Salem
Salim
Sedima
Segor
Seleucia Hisauriae
Sirie Celen
Socchoth
Sodomis
Syna
Tatnis
Taurum, mons
Tharso
Thebaida
Thesbe

En la mayoría de los casos se trata de nombres de regiones y/o provincias del Imperio Romano, ciudades o núcleos urbanos de diversa consideración, ríos, mares y en cuatro ocasiones, de lo que nosotras hemos denominado locus Egeriae. Estos locus Egeriae se definen por no entrar dentro de las categorías anteriormente citadas. Se trataría de Agrispecula (un campo baldío), cepos tu agiu Iohanni / hortus Sancti Iohannis (un huerto), Enon (una fuente o unas fuentes, el lugar donde bautizaba Juan), Memoriae concupiscentiae (conjunto de sepulcros en los alrededores del Monte Sinaí) y Opu Melchisedech (el lugar de Melquisedek, el sitio donde está la iglesia de Melquisedek).

Hemos procedido al análisis de cada topónimo, desde el punto de vista de la Etimología, la Lingüística, la Geografía y la Historia. Lamentablemente no hemos podido encontrar información lingüística y/o etimológica de todos los lugares mencionados en el viaje. Así, de los topónimos Arabot, Canaá, Dennaba, Fadana, Israhel,y Thesbe solo podemos presentar una información incompleta y poco precisa. Para la reconstrucción etimológica de cada topónimo, primero hemos consultado los estudios sobre el Itinerarium de los que disponíamos. Después hemos ampliado la búsqueda al Diccionario Enciclopédico de la Biblia y al Onomasticon de Perin. Después, y solo en aquellos casos donde las fuentes anteriores no hayan proporcionado ninguna información, hemos recurrido a las siguientes Enciclopedias: la Enciclopedia Italiana di Scienze, Lettere ed Arti y la Enciclopedia Universal Ilustrada.

Todos los topónimos de este artículo los denominaremos tal y como aparecen en el Itinerarium, esto es según su forma latina y con la flexión nominal en la que aparecen por primera vez.

\section{Clasificación y tipología de los topónimos}

Para la clasificación de este repertorio topográfico, hemos seguido la tipología que propone De Felice (2007:169-173), estableciendo una distinción entre una clasificación extralingüística, basada en las características y condiciones objetivas del lugar, y otra lingüística, basada en hacer una distinción lingüístico-formal de los topónimos.

Hemos ampliado este último grupo a una clasificación basada en la naturaleza del topónimo dentro de la lengua a la que pertenece, a partir del análisis que hace Gusmani (2007:87-114) de los fenómenos de Interferencia Lingüística.

A.-Análisis Extralingüístico: Hemos realizado cinco grupos, basándonos en los estudios de De Felice (2007:170-171), y un sexto, a partir de las condiciones particulares de nuestro repertorio:

Según la geografía del lugar, agrupando tanto los nombres relacionados con características morfológico-ambientales (geomórficas, geográficas y topográficas) como los relacionados con las condiciones biológicas (flora y fauna), o ambientales (utilidad). Es el grupo más numeroso. Dentro de este grupo encontramos los siguientes topónimos: Agrispecula, Asiam, Augustofratensis ${ }^{4}$ (provincia), Basan, Carneas, Chanaan (Terram), Choreb, Clesma, Edom, Egyptum, Enon, Eufraten / Eufrates, Fadana, Fenicis ("partes Fenicis"), Iordanem, Magdalum, Mansocrenas, Mesopotamiam, Mesopotamiam Syriae, Mortuum (mar), Nili, Oton, Pelusio, Persida, Rodanus, Rubrum (mar), Salim, Sedima, Segor, Sirie Celen, Socchoth, Tatnis, y Tauro/Taurum.

Los derivados de nombres étnicos: Arabia, Galatiam, Palestinam, y Thebaida.

Derivados del nombre del descubridor, del soberano, o de grandes personajes (políticos, científicos, literatos,...): Alexandriam (por Alejandro Magno), Anthiocia (en honor del Antíoco, padre de Seleuco I Nicator), Constantinopolim (por el emperador Constantino), Terra Gesse (por el personaje bíblico, Gessé), Helia (por el emperador Adriano-Aelius Hadrianus-), Israhel (por el sobrenombre de Jacob), Libiada (por Livia, esposa de Augusto), Terra Moab (por el personaje mítico de Moab), Opu Melchisedech (por el personaje bíblico de Melquisedek), Ponpeiopolim (por Cneus Pompeius Magnus), Ramessen (por el faraón Ramsés II), Seleucia Hisauriae (por el rey Seleúco I Nicator).

Denominaciones conmemorativas de hechos militares o relativos a la Historia nacional. También se incluyen en este grupo los nombres relacionados con ideologías políticas o, a nivel local, hechos o situaciones particulares de un núcleo pequeño. Así encontramos: Calcedona (por el lugar de fundación), Edessam (por una ciudad homónima en Macedonia), y Hero / Heroum ciuitas (por Pithona, la ciudad de los héroes).

Agiotopónimos (nombres religiosos relacionados con una divinidad, o porla existencia de un lugar de culto): Belsefon (por Baal Sephón), cepos tu agiu Iohanni / hortus Sancti Iohannis (por San Juan Bautista), Fogor (lugar del culto a Beth-BaalPe'or), Gerapolim /

4 El topónimo Augustofratense podría incluirse en el grupo 3, por la denominación de Augusto, pero
dado que este nombre no hace referencia a ningún emperador en particular, sino a que era provincia dado que este nombre no hace referencia a ningún emperador en particular, sino a que era provincia imperial, la hemos incluido en el grupo 1, por la referencia al río Éufrates. 
Ierapolim (por la Ciudad Santa: Hierápolis), lericho (por el dios Luna yrj), Ierusolima in Ierusolimam (por el dios ugarítico šlm, entre otras etimologías), Mons Nabau (por el dios babilonio Nabu), Parthenicum (Mar) por la referencia a Isis la Virgen (del griego

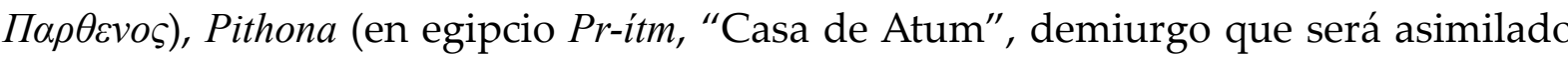
a Ra), Syna (por la divinidad lunar, Sin, entre otras etimologías), y Tharso (derivado del dios Tarku). Lamentablemente, tenemos un importante grupo de topónimos que no podemos incluir en ninguno de los grupos anteriores. Son los que hemos clasificado como "Desconocidos". Entre estos están: Mons Arabot, Regionem Ausitidem Batanis, Bithiniam, Cappadociam, Charris / Charra, Cilicia, Corico, Corra, Dennaba, Efesum, Epauleum. Esebon / Exebon,

Por el origen del topónimo. De Felice hace su clasificación en relación a una realidad geográfica objetiva (la toponimia de un país, una región, una provincia, una comarca una ciudad, ...). En cambio, en el Itinerium encontramos con dos realidades: una objetiva que coincide con parte de los territorios que recorre Egeria, y otra totalmente subjetiva, inspirada en la llamada geografía bíblica. Así, podemos clasificar los topónimos de Egeria en topónimos bíblicos y topónimos relacionados con el mundo grecolatino.

Topónimos Bíblicos: Incluimos en este grupo todos aquellos que Egeria menciona como relacionados con hechos incluidos en los relatos bíblicos. Entre ellos estarían Agrispecula, Anthiocia, Arabia, Montem Arabot, Regionem Ausitidem, Basan, Batanis, Belsefon, Bithiniam, Cappadociam, Carneas, cepos tu agiu Iohanni / hortus Sancti Iohannis, Terram Chanaan, Charris / Charra, Choreb, Cilicia, Corra, Dennaba, Edom, Efesum, Egyptum, Enon, Epauleum, Esebon / Exebon, Eufraten / Eufrates, Fadana, Faran, Fenicis ("partes Fenicis") Fogor, Galatiam, Gerapolim / Ierapolim, Terra Gesse, Hero / Heroum ciuitas, Hur, Idumeae, Iericho, Ierusolima, Iordanem, Israhel, Magdalum, Mesopotamiam, Terra Moab, Mare Mortuum, Montem Nabau, Nisibin, Opu Melchisedech, Oton, Palestinam, Pelusio, Persida Pithona, Ramessen, Mare Rubrum, Safdra, Salem, Salim, Sedima, Segor, Socchoth, Sodomis, Syna, Tatnis, Tharso, y Thesbe

Topónimos Grecolatinos: Son los que Egeria incluye para establecer el contacto entre la realidad del viaje que está realizando, esto es, la realidad que le circunda y la geografía bíblica que va siguiendo. En la mayoría de los casos se trata de las ciudades que pasa o ha pasado para ir de un lugar a otro (Alexandriam, Calcedona, Clesma, Constantinopolim, Corico, Edessam, Helia, Libiada, Mansocrenas, Nili, Ponpeiopolim, y Seleucia Hisauriae, ), o de la denominación de la realidad geopolítica romana (Asiam, Prouinciae Augustofratensis, Hisauria, Mesopotamiam Syriae, Mare Parthenicum, Rodanus, Sirie Celen, Montes Tauri, y Thebaida).

B.-Análisis lingüístico: Dos clasificaciones posibles: una desde el punto de vista de los sufijos más habituales en la formación de topónimos y otra en base a una distinción formal, entre topónimos simples y compuestos.
Sufijos: Solo cuatro topónimos presentan un sufijo: Prouincia Augustofratense, con el sufijo -ensis; Mare Parthenicum, con -enicum; y Persida y Thebaida, con -ida. Todos ellos, sufijos que forman parte del elenco latino habitual para la formación de palabras.

\section{Distinción formal:}

Simples: Un solo elemento léxico (o ya onomástico) con o sin sufijo: Alexandriam, Anthiocia, Arabia, Asiam, Basan, Batanis, Bithiniam, Calcedona, Cappadociam, Carneas, Charris / Charra, Choreb, Cilicia, Clesma, Corico, Corra, Dennaba, Edessam, Edom, Efesum, Egyptum,Enon, Epauleum, Esebon / Exebon, Eufraten / Eufrates, Fadana, Faran Fenicis, Galatiam, Hisauria, Hur, Idumeae, Iericho, Iordanem, Israhel, Libiada, Magdalum, Nili, Nisibin, Oton, Palestinam, Pelusio, Persida, Ramessen, Rodanus, Safdra, Salem, Salim, Sedima, Segor, Socchoth, Sodomis, Syna, Tatnis, Tharso, Thebaida, y

Compuestos: Formados por dos o más elementos, separados o juntos tanto a nivel hablado como en la grafía: Agrispecula, Montem Arabot, Prouinciae Augustofratensis, Regionem Ausitidem, cepos tu agiu Iohanni / hortus Sancti Iohannis, Terram Chanaan, Constantinopolim, Terra Gesse, Mansocrenas, Mesopotamiam, Mesopotamiam Syriae, Terra Moab, Mare Mortuum, Montem Nabau, Opu Melchisedech, Mare Parthenicum, Ponpeiopolim, Mare Rubrum, Seleucia Hisauriae, Sirie Celen, y Tauro. Dentro de este grupo, De Felice advierte de la posibilidad de encontrar tres casos particulares:

Los compuestos históricos (aquellos que eran nombres compuestos en origen pero que por la evolución de la lengua han perdido ese matiz). Entre los de Egeria estarían: Belsefon, Gerapolim / Ierapolim, Ierusolima, y

Los que por la multiplicidad de elementos en el nombre, se abrevian o simplifican: Fogor, Helia, y Hero.

Los tautológicos, es decir, los que presentan un mismo topónimo expresado de distintas maneras. De estos, no encontramos ningún caso dentro de nuestro repertorio.

Dado que, en general, podemos afirmar que los préstamos, aunque se presenten en gran cantidad, no implican que se produzcan modificaciones significativas en la estructura de la lengua receptora, ya que inciden casi exclusivamente en el léxico, y dado que, además, en nuestro caso se trata de un sector muy determinado del léxico del latín de Egeria, esto es, del repertorio toponímico, las generalizaciones que hagamos deberán ser completadas con un estudio exhaustivo de la lengua egeriana. Así pues, y establecida esta premisa, podemos afirmar que en el Itinerarium encontramos ante un cultural borrowing, dada la abundancia de préstamos que hemos percibido. Aunque intuimos (sabemos), que la realidad en la que se mueve nuestra peregrina está más cerca de un intimate borrowing, donde el uso del griego desde hace ya varios siglos habrá influido en la forma de hablar y escribir de las gentes del siglo IV. Es más, casi estamos seguras de poder afirmar que nos encontramos ante un fenómeno de disglosia, 
donde el griego, como hemos podido comprobar anteriormente, sería la lengua de comunicación, por excelencia, en ambientes extranjeros (Gusmani, 2007: 109-110) Todo ello sobre un importante sustrato de lenguas anteriores, que en este caso serían el hebreo y el egipcio.

\section{Conclusione}

No somos las primeras en acercarnos al estudio de la Toponimia. La Toponimia, según la definición del profesor De Felice es il settore delle scienze onomastiche che

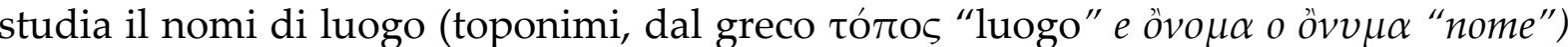
(De Felice, 2007:164)

En los nombres de lugar encontramos un resumen, un reflejo (Rohlfs, 1985:2555 y Terrado, 1999:24-26), de la historia de un municipio, de una provincia, de una región. Su objetivo es el estudio de los nombres de lugar, desde el punto de vista de la Lingüística (Terrado, 1999:16-26 y De Felice, 2007:164). Así hasta aquí tenemos dos hechos objetivos - la existencia de nombres de lugar, topónimos, que sirven para identificar un espacio geográfico y la utilización que desde diversas parcelas del saber se hace de estos topónimos - $y$, junto a ellos, un principio sobre el que se asienta dicha utilización: el topónimo se entiende como la suma de un significante y un significado, siendo este último el que realmente tiene valor para las ciencias extralingüísticas. Ocurre sin embargo que en la mayor parte de las ocasiones todo ese cúmulo de información se nos presenta no directamente, sino cifrado, en clave, sólo si se interpreta adecuadamente esa clave, es posible acceder a la información que se esconde tras el nombre. En efecto, lo que a primera vista encontramos en los topónimos es únicamente una secuencia fónica que sirve para identificar un espacio geográfico dado. Se trata entonces de un elemento lingüístico y, en cuanto tal, ha de ser analizado desde presupuestos estrictamente lingüísticos si queremos extraer de manera fiable la información que dicho elemento encierra (Morala, 1986:50).

Estos topónimos se refieren tanto a los nombres relacionados con los elementos geográficos naturales (continentes, islas, penínsulas, montañas y otros efectos del relieve, llanuras, y formas relacionadas con el terreno, océanos, mares, golfos, bahías, estrechos, lagos, ríos, y toda masa de agua,...) y /o los relacionados con estructuras y elementos creados por el ser humano (estados y regiones históricas, ciudades, países, cualquier núcleo habitado sin importar su tamaño, grandes vías de comunicación, calles, plazas, cualquier elemento urbano, puentes, viaductos, túneles, canales, embalses y pantanos artificiales, pozos, cisternas, puertos y muelles, construcciones agrícola-pastoriles, artesanales o industriales,...) (De Felice, 2007:164).

5 «...la parte de la ciencia Onomástica que estudia los nombres de lugar (los topónimos del griego

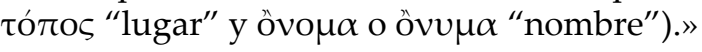

A través del estudio de estos topónimos se pretende, en el plano diacrónico, la reconstrucción del origen del étimo como resultado de la interacción de quienes han vivido en la zona y el signo (léxical o ya onomástico), en que lo manifiestan y sus cambios y transformaciones, e incluso de la sustitución del mismo, a lo largo del tiempo. En el plano sincrónico, se pretende estudiar su difusión en un área determinada en el tiempo y el espacio, y su frecuencia absoluta y relativa. Los métodos para realizar estos estudios son múltiples, aunque hemos de resaltar las dos formas más básicas de acercamiento al estudio toponímico: el método histórico-filológico y el tipológico (De Felice 2007:164-165). Nosotros emplearemos ambos métodos, pero dentro de la tipología nos orientaremos en dos direcciones distintas: por un lado, un análisis formal, por otro, un análisis estructural, a partir de la lengua primaria de Egeria.

Las características fundamentales de la Toponimia (De Felice 2007:165-167), y que en la Peregrinatio vemos perfectamente reflejadas, son

Una ingente cantidad de topónimos. Cuestión fácil de imaginar si tenemos en cuenta el tamaño del globo terráqueo y la cantidad de lugares que podemos apreciar, o el viaje que se ha de emprender. Egeria cruzó y visitó todo el mundo conocido, desde la zona de Gallaecia, la Galia o Normandia hasta el Oriente Próximo.

Su conservación a lo largo del tiempo y de los cambios étnicos o culturales, mutando en muchas ocasiones y por los avatares de la evolución de la lengua, pero sin perder de vista el étimo. Entre el Iericho de Egeria y nuestro Jericó hay más de mil seiscientos años, pero la raíz no ha cambiado.

La existencia de uno o más topónimos para un mismo lugar (De Felice 2007:166167). Este fenómeno se produce por tres razones fundamentales: la existencia de áreas de poliglosia o polilingüismo, la convivencia entre el nombre dialectal y el oficial, y que se den nombres distintos al mismo lugar en las zonas costeras (uno dado por las gentes de tierra y otros por las del mar). En el Itinerarium es una característica que encontramos con relativa frecuencia: Basan o Safdra, Carneas o Dennaba, Charris o Charra, Esebon o Exebon, Selim o Sedima, Pithom o Heroópolis,...

El primer resultado de un estudio toponímico es la creación de un repertorio (De Felice 2007:167-169), esto es, el conjunto de los topónimos existentes en un momento cronológico concreto en un área determinada. Este área puede ser determinada por razones históricas, político-administrativas, lingüísticas y culturales y/o étnicas. En nuestro caso concreto, y forzando un poco la definición que da De Felice (2007:167), el repertorio toponímico objeto de estudio lo forman el conjunto de topónimos que utiliza Egeria en la primera parte de su Itinerarium, donde narra sus viajes. En la llamada segunda parte, Egeria se centrará en describirnos la liturgia de Jerusalén. Su análisis, 
desde el punto de vista diatópico, se saldría de lo planteado en este estudio, pero lo abordaremos en el futuro.

\section{REFERENCIAS BIBLIOGRÁficAS}

Alturo, J., “Deux nouveaux fragments de l'Itinerarium Egeriae” du Ixe-X siècle”, Revue Bénédictine, 115 (2005), pp. 241-250.

Arce, A., Itinerario de Egeria 381-384, Madrid, BAC, 1980 (19962; 2010³).

Arias Abellán, C., Itinerarios latinos a Jerusalén y al Oriente Cristiano. Egeria y el Pseudo-Antonino de Piacenza, Sevilla, Secretariado de Publicaciones de la Universidad de Sevilla. 2000.

Bertini, F.; Cardini, F.; Fumagalli Beonio Brocchieri, M. T.; Leonardi, C., Medioevo al femminile, Roma - Bari, 1989.

Bruyne, D. de, “Nouveaux fragments de l'Itinerarium Eucheriae”, Revue Benedictine, 26 (1909), pp. 481-484.

Cardini, F., “Egeria la pellegrina», en Bertini-Cardini-Fumagalli-Beonio Brocchieri-Leonardi (1989: 3-38).

Cruz Herranz, L. M. De La,“O manuscrito do Itinerarium Egeriae”, en Novoa (2003: 105-123).

De Felice, E, “Onomástica”, en Lazzeroni (2007: 147-179).

Diccionario Enciclopédico de la Biblia, Barcelona, Ed. Herder, 1993.

Enciclopedia Italiana di Scienze, Lettere ed Arti, Istituto della Enciclopedia Italiana, 1949.

Enciclopedia Universal Ilustrada, Madrid, Espasa Calpe, 1980.

Gusmani, R. (2007): “Interlinguistica”, en Lazzeroni (2007: 86-114)

Janeras, S., Egèria. Diari d'un pelegrinatge a Terra Santa, Barcelona, Centre de Pastoral Litúrgica, 2010

-, Égeria. Peregrinatge, Barcelona, Fundació Bernat Metge, 1986

Lazzeroni, R. (ed.), Linguistica storica, Roma, Carocci Editore, 2007.

Milani, C., "I grecismi nell'Itinerarium Egeriae”, Aevum, 43 (1969), pp. 200-234.

Morala Rodríguez, J.R., “El nombre propio ¿Objeto de estudio interdisciplinar?”, Contextos, IV/8 (1986), pp. 49-61

Natalucci, N., Pellegrinaggio in Terra Santa (Itinerarium Egeriae), Florencia, Biblioteca Patrística, 17,1991

Novoa, F. (dir.), De Fisterra a Xerusalén. Exeria e os primeiros peregrinos cristiáns, Santiago de Compostela, Museo das Peregrinacións, 2003.

Perin, J. et alii, Lexicon totius latinitatis, Bologna: Forni, Tomos Onomasticon A-K y Onomastikon J-Z, 1965

Rohlfs, G., Antroponimia e Toponomastica nelle lingue neolatine. Aspetti e Problemi, Tubinga, 1985.
Swanson, D. C., "A Formal Analysis of Egeria's (Silvia' s) Vocabulary", Glotta, 44 (1966/67), pp. 177-254.

Terrado Pablo, J., Metodología de la investigación en Toponimia, Lérida (Pintor Morena y Galicia, 4): J. Terrado, Zaragoza, 1999. 